\title{
Conhecimento de Mulheres Incontinentes sobre Incontinência Urinária: uma Reflexão Teórico-Crítica
}

\author{
Knowledge of Incontinent Women about Urinary Incontinence: \\ a Theoretical and Critical Reflection
}

\author{
Conocimientos de las Mujeres Incontinentes acerca de la Incontinencia \\ Urinaria: una Reflexión Teórica y Crítica
}

Claudia Daniella Avelino Vasconcelos Benício', Maria Helena Barros Araújo Luz', Nirvânia do Vale Carvalho², Bianca Anne Mendes de Brito', Jose Lucas Silveira Ferreira'

\begin{abstract}
RESUMO
Introdução: A incontinência urinária é a perda involuntária de urina, e pode ocasionar problemas físicos, emocionais, sociais e econômicos. Objetivo: Despertar nos profissionais de saúde, bem como nos gestores de saúde, a dimensão da problemática referente à incontinência urinária. Métodos: Trata-se de uma reflexão teórico-crítica oriunda dos resultados de uma dissertação de mestrado do Programa de Pós-graduação (Mestrado) em Enfermagem da Universidade Federal do Piauí sobre a falta de conhecimento por parte das mulheres com incontinência urinária quanto à referida condição. Resultados: O conhecimento destas mulheres sobre esta problemática é insuficiente para promover o autocuidado e/ou buscar auxílio profissional. Conclusão: Faz-se necessário discutir sobre as possibilidades de informação e de cuidados que podem ser dispensados às mulheres incontinentes, por meio de uma busca teórico-prática dos principais tipos de tratamento para tal enfermidade, bem como refletir sobre as ações que os profissionais de saúde, os órgãos públicos e os veículos de comunicação têm feito e podem fazer em prol da melhoria da qualidade de vida das mulheres incontinentes.
\end{abstract}

DESCRITORES: Estomaterapia. Conhecimento. Incontinência urinária. Mulheres.

\begin{abstract}
Introduction: Urinary incontinence is the involuntary loss of urine that may result in physical, emotional, social, and economic problems. Objectives: To awaken health professionals and health managers to the dimension of the urinary incontinence problematics. Methods: This is a theoretical and critical reflection based on the results of a master's thesis of a Graduate Master Program in Nursing from University Federal do Piaui about lack of knowledge by women with urinary incontinence with regard to this condition. Results: Knowledge of incontinent women on urinary incontinence is insufficient to promote self-care and/or seek professional help. Conclusion: Discussion about the possibilities of information and care provided to incontinent women through a theoretical and practical search of the major types of treatment for such disease is necessary. In addition, we should reflect on the actions that health professionals, public bodies, and communication vehicles have done and can do to improve the quality of life of incontinent women.
\end{abstract}

DESCRIPTORS: Stomatherapy. Knowledge. Urinary incontinence. Women.

'Programa de Pós-graduação (Mestrado e Doutorado) em Enfermagem da UFPI - Teresina (PI), Brasil.

Endereço para correspondência: Avenida Universitária, s/n - Campus Universitário Ministro Petrônio Portela - bloco 12 - CEP: $64049-550$ -

Teresina (PI), Brasil - E-mail: cdavb2011@yahoo.com.br

${ }_{2}^{2}$ Mestre em Enfermagem, Enfermeira Intensivista da UTI do Hospital Universitário do Piauí e do Hospital Getúlio Vargas - Teresina (PI), Brasil.

Artigo recebido em: 22/04/2015 - Aceito para publicação em: 17/04/2016 


\section{RESUMEN}

Introducción: La incontinencia urinaria es la pérdida involuntaria de orina, que puede causar problemas físicos, emocionales, sociales y económicos. Objetivos: Despertar en los profesionales de salud y gestores de salud la dimensión de la problemática referente a la incontinencia urinaria. Métodos: Se trata de una reflexión teórica y crítica de los resultados de una tesis del Programa de Posgrado (Maestro) en Enfermería de la Universidad Federal de Piauí sobre la falta de conocimiento por mujeres con incontinencia urinaria sobre esta condición. Resultados: El conocimiento de esas mujeres sobre este problema es insuficiente para promover el auto-cuidado y/o buscar ayuda profesional. Conclusión: Es necesario discutir sobre las posibilidades de información y atención que pueden ser suministrados a las mujeres incontinentes, por medio de la búsqueda teórico y práctica de los tipos principales de tratamiento para esta enfermedad, y reflexionar sobre las acciones que los profesionales de salud, organismos públicos y vehículos de comunicación han hecho y pueden realizar hacia la mejora de la calidad de vida de las mujeres incontinentes.

PALABRAS CLAVE: Conocimiento. Incontinencia urinaria. Mujeres.

\section{INTRODUÇÃO}

A incontinência urinária (IU) é definida pela International Society of Continence e pela International Urogynecological Association como a queixa de qualquer perda involuntária de urina, e não raramente pode desencadear prejuízos à vida cotidiana das mulheres, principalmente isolamento social, depressão, problemas físicos e de ordem econômica ${ }^{1}$. É uma questão que tem sido pauta de discussões entre alguns profissionais de saúde, tais como enfermeiros, médicos, fisioterapeutas, psicólogos, dentre outros, os quais se preocupam em oferecer uma melhor qualidade de vida às pessoas que lidam com esta doença.

Segundo Buckley e Lapitan ${ }^{2}$, a prevalência de IU em todo o mundo varia entre 25 e 45\%. Outros estudos evidenciaram uma média de IU de $27,6 \%$ no sexo feminino e $10,5 \%$ no masculino. Tal enfermidade se faz presente em aproximadamente 20 a $23 \%$ das mulheres na faixa etária dos 30 aos 39 anos, aumentando para 25 a 30\% dos 40 a 49 anos, mantendo-se assim até os 75 a 89 anos, quando ocorre um novo crescimento para 30 a 35\%, e após os 90 anos, a prevalência chega a $35 \%{ }^{3}$. Assim, há um aumento diretamente proporcional à idade, que pode atingir até 50\% das mulheres em alguma fase de suas vidas ${ }^{2,3}$.

A IU é um agravo da saúde de incidência e prevalência crescentes, especialmente em mulheres, sendo o tipo mais comum a incontinência urinária de esforço (IUE), ou seja, quando a perda de urina ocorre por esforços, exercícios, espirro ou tosse. A IUE está associada a quase $50 \%$ dos casos, pois além da anatomia feminina favorecer esta ocorrência, as alterações hormonais e os efeitos provocados por gestações e partos podem propiciar o deslocamento e enfraquecimento dos músculos perineais ${ }^{4}$.
Desse modo, percebe-se que a problemática da IU é mais frequente em mulheres e, dentre estas, podem ser observados como fatores de risco associados à sua ocorrência: diabetes melitus; medicações anti-hipertensivas; tabagismo; doenças pulmonares, como a doença pulmonar obstrutiva crônica; gestações e partos; alterações hormonais; infecções do trato urinário; sobrepeso; cirurgias pélvicas e, para alguns pesquisadores, ainda não comprovado cientificamente, a atividade física de alto impacto associada ao esforço físico ${ }^{5-7}$.

No entanto,já existem vários tipos de terapias adotadas para a melhoria e até mesmo cura da incontinência urinária, como o tratamento conservador com o uso de fármacos, os exercícios que podem melhorar a sustentação e a tonicidade da musculatura pélvica e perineal, a cinesioterapia, a eletroestimulação, o biofeedback, os cones vaginais, os tubos, a terapia comportamental e as cirurgias. Salienta-se que propostas de atividades físicas podem ser desenvolvidas no sentido de melhorar a continência, nas quais são incluídos exercícios de fortalecimento do assoalho pélvico ${ }^{8,9}$, que podem ser realizados até mesmo em domicilio, uma vez previamente orientados por um profissional especializado.

Portanto, objetiva-se com esta reflexão despertar nos profissionais de saúde, bem como nos gestores de saúde, a dimensão da problemática da IU.

\section{MÉTODOS}

Trata-se de uma reflexão teórico-crítica oriunda dos resultados de uma dissertação de mestrado do Programa de Pós-Graduação (Mestrado) em Enfermagem da Universidade Federal do Piauí acerca da falta de conhecimento, por parte das mulheres com IU, relacionado à referida condição. 
Além disso, em função da inquietação da primeira autora diante da experiência enquanto enfermeira por sete anos em clínica especializada de Urologia, onde se evidenciou a desinformação da demanda feminina com IU com relação ao problema com o qual conviviam, emergiram questionamentos que serão abordados nos resultados.

\section{RESULTADOS E DISCUSSÃO}

\section{Por que muitas mulheres não conhecem os tratamentos para a incontinência urinária?}

Apesar do conhecimento científico produzido e das tecnologias desenvolvidas na tentativa de minimizar as consequências provocadas pela IU, acredita-se que as informações e os métodos para a sua correção ainda não foram veiculados a ponto de repercutirem positivamente na saúde da população feminina incontinente, pois, de acordo com os resultados encontrados na pesquisa em questão, mais de $50 \%$ das mulheres participantes do estudo ( $\mathrm{n}=306)$ não conheciam os diversos aspectos da IU, como suas causas, seus tipos e seu tratamento, os quais seriam adequados e talvez suficientes para melhorar a continência. Diante desses fatos, questiona-se por que grande parte das mulheres não tem conhecimento sobre a IU e os seus tipos de tratamento?

Vivemos atualmente em um mundo globalizado onde as informações são veiculadas de forma instantânea, principalmente às pessoas que possuem algum tipo de acesso aos veículos de comunicação. As políticas de saúde têm sido implantadas e implementadas com o propósito de beneficiar a comunidade, contribuindo com a identificação e o tratamento de agravos que incidem e prevalecem em meio à quantidade enorme de desinformação (e a IU é um deles). Mesmo assim, é notória a inércia tanto das pessoas acometidas pela IU como dos profissionais no sentido de trazer à tona este silencioso problema que é mascarado pelo descaso, pela omissão e acomodação de ambas as partes. Seria o constrangimento das mulheres em não querer se expor a principal razão para tal? Ou talvez a "falta de tempo", a "dificuldade de investigar" ou até mesmo o "despreparo" dos profissionais em querer ou poder explorar melhor esta queixa?

Por estes e talvez por outros motivos, a IU muitas vezes é subnotificada, pois o estereótipo que a doença traz consigo dificulta o seu diagnóstico e, por conseguinte, o tratamento de muitas pessoas que sofrem com a incontinência, as quais preferem se ocultar frente à problemática.

\section{Refletindo o acesso às informações sobre a incontinência urinária}

Baseando-se em tais questões, propõe-se uma reflexão quanto à necessidade de acessibilidade às informações sobre este problema de saúde de repercussões tão negativas à vida das pessoas. Questiona-se por que não sugerir ou idealizar uma campanha nacional de divulgação para prevenir e promover a saúde das pessoas que vivenciam a IU? Por que pouco se veicula na mídia sobre o problema? Não seria oportuno a IU estar presente de maneira enfática nos históricos de saúde das instituições para que se busque tal diagnóstico no momento em que admitimos ou avaliamos um(a) paciente? Acredita-se que, dessa forma, seria possível dar atenção a um problema que tem provado a sua dimensão, apesar de ser apenas mostrado por trás de discursos não espontâneos de pacientes que convivem com o problema, relatados por meio de entrevistas direcionadas como pesquisas e trabalhos de campo realizados por profissionais que insistem e não desistem em tentar demonstrar que a IU é um problema que precisa ser pelo menos reconhecido como uma doença.

Muitas pesquisas já foram desenvolvidas e publicadas em periódicos nacionais e internacionais, produzidas por profissionais diversos da área de saúde, tais como: enfermeiros, médicos, fisioterapeutas e psicólogos. Talvez estes sejam os mesmos que se deparam muitas vezes com a clientela feminina incontinente durante suas avaliações de saúde em consultas e até mesmo em internações. Assim, acredita-se que algo benéfico em relação à continência é socializado por tais profissionais entre as pessoas acometidas por este problema, pois se encontram exceções, ou seja, mulheres conhecedoras de sua problemática (a IU), o que chega a ser surpreendente quando nos deparamos com uma mulher que conhece algo sobre seu problema.

No entanto, ainda é perceptível, na vivência da primeira autora, o subjulgamento da IU por outros profissionais de saúde que, mesmo inseridos nesse contexto, não a valorizam como problema de saúde, passível de provocar alterações que variam desde físicas a graves problemas emocionais. Infelizmente, muitos especialistas do nosso meio ainda desconhecem que aproximadamente 200 milhões de 
pessoas no mundo apresentam algum tipo de IU, e que uma em cada quatro mulheres com idades entre 30 e 59 anos já vivenciou algum episódio de $\mathrm{IU}^{10}$.

\section{CONCLUSÃO}

A desinformação provocada pelos diversos fatores citados nesta reflexão parece ser o obstáculo para a sensibilização e consequentemente para o início da tomada de consciência do problema da IU por parte das mulheres incontinentes. No entanto, o medo de expor seus sentimentos, a sua dignidade e o seu valor perante o profissional e até mesmo a sociedade termina por torná-las omissas frente à perda do controle urinário, comprometendo a autonomia do funcionamento de seu próprio corpo, o que repercute muitas vezes na não aceitação do companheiro, na sua sexualidade, autoimagem e autoestima, desencorajando a sua busca por ajuda e se recusando ao desafio de enfrentar as dificuldades na luta por medidas de prevenção, tratamento e cura da IU.

Dessa forma, a desvalorização das queixas apresentadas por estas mulheres contribui significativamente para que o problema não seja percebido na prática por elas e consequentemente pelo profissional de saúde que poderia reconhecê-lo e indicar algum tipo de tratamento adequado.

Assim, ao final desta reflexão, pode-se sugerir que o desenvolvimento de estratégias ou políticas de saúde voltadas para a problemática em questão seriam, talvez, os meios favoráveis para despertar a atenção das mulheres e dos profissionais de saúde para a problemática da IU, possibilitando desta forma uma melhor inserção destes no sentido de investigar, diagnosticar, orientar e tratar a IU.

Acredita-se que, assim, os prejuízos provocados pela IU à vida das mulheres poderiam ser amenizados, contribuindo para a melhoria da qualidade de vida delas e a redução dos índices de incidência e prevalência da IU.

\section{REFERÊNCIAS}

1. Abrams P, Cardozo L, Khoury Saad, Wein Alan. Incontinence. 5th International Consultation on Incontinence. Paris: ICUD-EAU; 2012.

2. Buckley BS, Lapitan MC. Prevalence of urinary incontinence in men, women, and children - current evidence: findings of the Fourth International Consultation on Incontinence. Urology. 2010;76(2):265-70.

3. Minassian VA, Drutz HP, Al-Badr A. Urinary incontinence as a worldwide problem. Int J Gynaecol Obstet. 2003;82(3):327-38.

4. Seim A, Eriksen BC, Hunskarr S. A study of female urinary incontinence in general practice. Demography medical history, and clinical findings. Scand J Urol Nephrol. 1996;30(6):465-71.

5. Higa R, Lopes MH, Reis MJ. Fatores de risco para incontinência urinaria na mulher. Rev Esc Enferm USP. 2008;42(1):187-92.
6. Danforth KN, Townsend MK, Lifford K, Curhan GC, Resnick NM, Grodstein F. Risk factors for urinary incontinence among middle-aged women. Am J Obstet Gynecol. 2006;194(2):339-45.

7. Guarisi T, Pinto Neto AM, Osis MJ, Pedro AO, Paiva LH, Faúndes A. Incontinência urinária entre mulheres climatéricas brasileiras: inquérito domiciliar. Rev Saúde Pública. 2011;35(5):428-35.

8. Chiapara TR, Cacho DP, Alves AF. Incontinência urinária feminina: assistência fisioterapêutica e multidisciplinar. São Paulo: Livraria Médica, Paulista Editora; 2007.

9. Caetano AS, Tavares MC, Lopes MH, Poloni RL. Influência da atividade física na qualidade de vida e auto-imagem de mulheres incontinentes. Rev Bras Med Esporte. 2009;15(2):93-97.

10. Contregas 00 . Stress urinary in ginecological pratice. Int J Gynaecol Obstet. 2004;86(1):6-16. 